\title{
Índice de segurança técnica da equipe de enfermagem da pediatria de um hospital de ensino*
}

ÍNDICE DE SEGURIDAD TÉCNICA DEL EQUIPO DE ENFERMERÍA DE LA PEDIATRÍA DE UN HOSPITAL DE ENSEÑANZA

\author{
Karin Emilia Rogenski', Fernanda Maria Togeiro Fugulin"
}

\section{RESUMO}

O estudo teve por objetivo identificar o Índice de Segurança Técnica (IST) da equipe de enfermagem da Unidade de Pediatria do Hospital Universitário da USP, no período de 2001 a 2005. Os percentuais de cada tipo de ausência, dos profissionais de enfermagem, foram obtidos junto ao Departamento de Enfermagem ou calculados com base nas equações propostas na literatura. O percentual de ausências por folgas correspondeu ao maior percentual de cobertura dos trabalhadores de enfermagem. Os percentuais totais de ausências não previstas foram inferiores àqueles referentes às ausências previstas, contrariando o discurso das enfermeiras que relacionam esse tipo de ausência como o principal responsável pela insuficiência de pessoal nas instituições de saúde. A variação dos ISTs apontou a importância de serem realizadas avaliações sistemáticas das ausências da equipe de enfermagem, assim como de se identificarem índices específicos das unidades, como subsídio para a avaliação do quadro de pessoal nas organizações de saúde.

\section{DESCRITORES}

Absenteísmo.

Equipe de enfermagem.

Recursos humanos de

enfermagem no hospital.

\section{ABSTRACT}

This study was aimed at identifying the Technical Safety Index (ITS, in the Portuguese acronym) of the nursing team of the University of São Paulo’s Hospital Universitário's Pediatrics Unit (20012005). Percentages for each type of absence of nursing professionals were obtained from the Nursing Department or calculated based on the equations found in the literature. Absences due to days off were the highest percentage. Total percentages of unplanned absences were lower than those referring to planned absences, which contradict nurses who report this type of absence as the main cause for health institutions understaffing. IST variation highlights the importance of carrying out systematic evaluations of the nursing team's absences, as well as of identifying specific indexes for each unit, as subsidies for the evaluation of the personnel in health organizations.

\section{KEY WORDS}

Absenteeism.

Nursing, team.

Nursing staff, hospital.

\section{RESUMEN}

El estudio tuvo por objetivo identificar el Índice de Seguridad Técnica (IST) del equipo de enfermería de la Unidad de Pediatría del Hospital Universitario de USP, en el periodo de 2001 a 2005. Los porcentajes de cada tipo de ausencia, de los profesionales de enfermería, fueron obtenidos junto al Departamento de Enfermería o calculados con base en las ecuaciones propuestas en la literatura. El porcentaje de ausencias por descanso correspondió al mayor porcentaje de cobertura de los trabajadores de enfermería. Los porcentajes totales de ausencias no previstas fueron inferiores aquellos referentes a las ausencias previstas, contrariando el discurso de las enfermeras que relacionan ese tipo de ausencia como el principal responsable por la insuficiencia de personal en las instituciones de salud. La variación de los ISTs apuntó la importancia de que sean realizadas evaluaciones sistemáticas de las ausencias del equipo de enfermería, así como de que se identifiquen índices específicos de las unidades, como subsidio para la evaluación del cuadro de personal en las organizaciones de salud.

\section{DESCRIPTORES}

Absentismo.

Grupo de enfermería.

Personal de enfermería en hospital.

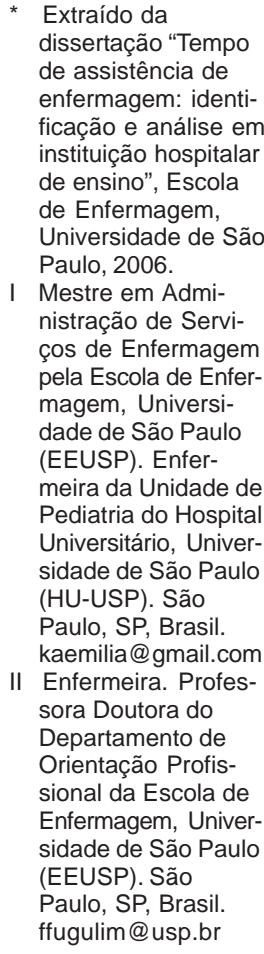

I Mestre em Administração de Serviços de Enfermagem pela Escola de Enfermagem, Universidade de São Paulo (EEUSP). Enfermeira da Unidade de Pediatria do Hospita Universitário, Universidade de São Paulo (HU-USP). São Paulo, SP, Brasil. kaemilia@gmail.com

II Enfermeira. Professora Doutora do Departamento de Orientação Profissional da Escola de Enfermagem, Universidade de São Paulo (EEUSP). São Paulo, SP, Brasil. ffugulim@usp.br 


\section{INTRODUÇÃO}

As organizações de saúde, dentre elas as hospitalares, tem investido na busca de novas estratégias de gestão que possibilitem conciliar a redução dos custos, a melhoria da qualidade dos serviços oferecidos e a satisfação dos clientes.

Diante desse cenário, a temática dimensionamento de pessoal de enfermagem assume importante significado, na medida em que procura adequar o quadro de pessoal disponível às necessidades assistenciais da clientela, aos objetivos institucionais e às expectativas dos clientes internos e externos.

Dentre as principais variáveis intervenientes nos métodos de dimensionamento de pessoal de enfermagem, a determinação de um índice para a cobertura das ausências dos trabalhadores ao serviço constitui-se um aspecto extremamente importante, em decorrência das implicações que a redução da equipe de enfermagem acarreta na quantidade e na qualidade da assistência prestada ao paciente, especialmente nas unidades que funcionam ininterruptamente ${ }^{(1)}$.

As ausências na enfermagem desorganizam o trabalho da equipe. A ausência de um elemento traz graves perturbações à realização das atividades e sobrecarga aos demais membros do grupo. Reduz a produção, aumenta o custo operacional e dificulta a substituição dos trabalhadores diretamente ligados à assistência, diminuindo sensivelmente a qualidade do cuidado prestado.

Entende-se como ausências previstas os dias relativos às folgas (descanso semanal remunerado e feriado não coincidente com o domingo) e às férias; como ausências não previstas os dias relativos às faltas, às licenças e às suspensões ${ }^{(2)}$.

A expressão Índice de Segurança Técnica (IST) referese a um acréscimo percentual no quantitativo de pessoal de enfermagem, por categoria profissional, para a cobertura de todos esses tipos de ausências.

Dessa forma, o conhecimento do comportamento dos trabalhadores em relação a essa variável e o estabelecimento de índices compatíveis com cada realidade, determinam a quantidade de trabalhadores que devem ser acrescentados ao número total de funcionários de uma dada categoria profissional para a cobertura dessas ausências, bem como as medidas necessárias para conter os índices encontrados ${ }^{(1)}$.

O percentual a ser acrescentado sobre o total dos componentes da equipe de enfermagem, destinados à cobertura dessas ausências, varia entre os autores que desenvolveram estudos sobre esta temática ${ }^{(1-8)}$.
A Resolução n ${ }^{\circ}$ 293/04 $4^{(9)}$, do Conselho Federal de Enfermagem (COFEN), estabelece que o IST não deve ser inferior a 15\%, dos quais 8,33\% referem-se à cobertura das ausências por férias e 6,67\% à cobertura das ausências não previstas (valor empírico), uma vez que as ausências relativas às folgas já estão consideradas na metodologia utilizada para o dimensionamento de pessoal de enfermagem. Preconiza, ainda, que nas unidades de internação, onde o quadro de profissionais de enfermagem possui $60 \%$ ou mais de pessoas com idade superior a 50 anos, deverá ser acrescido $10 \%$ ao IST. As unidades deverão dispor, também, de 3 a $5 \%$ do quadro geral de profissionais de enfermagem para cobertura de situações relacionadas à rotatividade de pessoal e participação em programas de Educação Continuada.

Estudo realizado nas unidades de internação de um hospital de ensino, sobre o percentual de ausências previstas e não previstas dos trabalhadores de enfermagem, evidenciou uma variação entre os índices encontrados em cada uma das unidades e indicou a possibilidade de estar ocorrendo sobrecarga de trabalho, em decorrência do número de ausências, em algumas unidades. As autoras concluíram que a identificação do percentual de ausências da equipe de enfermagem evidenciou a arbitrariedade em se utilizar os índices de cobertura indicados na literatura, tendo em vista a disponibilidade de métodos que possibilitam a identificação desses valores, de acordo com a realidade de cada instituição ${ }^{(1)}$.

Diante do exposto, considerando a importância e a influência dessa variável no quantitativo de pessoal de enfermagem, optou-se pela realização dessa pesquisa, que teve por objetivo identificar o IST da Unidade de Pediatria do Hospital Universitário da Universidade de São Paulo (HU-USP), no período de 2001 a 2005.

\section{MÉTODO}

O estudo, de abordagem quantitativa, do tipo exploratório-descritivo, foi desenvolvido na Unidade de Pediatria do HU-USP, no período de janeiro de 2001 a dezembro de 2005.

Para identificar o IST da Unidade de Pediatria do HUUSP, no período de 2001 a 2005, partiu-se da equação:

$$
I S T_{k} \%=\left\{\left[\left(1+\frac{R_{k} \%}{100}\right) \cdot\left(1+\frac{V_{k} \%}{100}\right) \cdot\left(1+\frac{A_{k} \%}{100}\right)\right]-1\right\} \cdot 100
$$

onde:

$k$ = categoria profissional;

$R_{k} \%$ = percentual de ausência por folga semanal e feriado não coincidente com o domingo, da categoria profissional ${ }_{k}$; 
$V_{k} \%=$ percentual de ausência por férias anuais, segundo a categoria profissional $k$;

$A_{k} \%=$ percentual de ausência não prevista, segundo a categoria profissional ${ }_{k}$.

Os percentuais de cada tipo de ausência, dos profissionais de enfermagem da Unidade de Pediatria do HU-USP, referentes aos anos 2003 e 2004, foram obtidos junto ao Departamento de Enfermagem (DE). Para os demais períodos do estudo, os percentuais referentes a cada tipo de ausência dos profissionais de enfermagem, foram calculados a partir das equações ${ }^{(10)}$ demonstradas a seguir.

Entretanto, verificando-se que, na prática profissional, não há diferença entre as atividades realizadas pelos técnicos e auxiliares de enfermagem, decidiu-se agrupar as duas categorias a fim de estabelecer um percentual médio de ausências único, referente aos profissionais de nível médio.

\section{Ausência prevista por folga semanal e feriado não coincidente com o domingo ( $\left.\mathrm{R}_{\mathrm{k}} \%\right)$}

O método de dimensionamento de pessoal de enfermagem propõe uma equação específica para determinar do percentual de ausência por folga semanal remunerada e outra para feriados não coincidentes com o domingo ${ }^{(10)}$. No entanto, considerando que o número de dias de folga indicadas nas escalas mensais da Unidade de Pediatria do HU-USP, onde os dados foram levantados, referem-se tanto às folgas semanais remuneradas, quanto àquelas concedidas por feriados não coincidentes com o domingo, optou-se por estabelecer um percentual de ausências único, referente aos dois tipos de ausências previstas (folga semanal remunerada e folga por feriado não coincidente com o domingo).

Este percentual foi calculado por meio da seguinte equação:

$$
R_{k} \%=\left(\frac{r_{k}}{d-r_{k}}\right) \cdot 100
$$

onde :

$k=$ categoria profissional

$R_{k} \%$ = percentual de ausências por folga semanal remunerada e feriados não coincidente com o domingo, da categoria ${ }_{k}$;

$r_{k}=$ número médio de dias de folga, dos trabalhadores de enfermagem, da categoria $_{k}$;

$d=$ Dias do mês.

\section{Ausências previstas por férias $\left(\mathrm{V}_{\mathrm{k}} \%\right)$}

A aplicação da equação ${ }^{(10)}$, apresentada a seguir, possibilitou a identificação do percentual de ausências previstas por férias:

$$
V_{k} \%=\frac{v k}{D-v k} \cdot 100
$$

onde:

$k=$ categoria profissional

$V_{k} \%=$ percentual de ausências por férias anuais, da categoria profissional $_{k}$;

$v_{k}=$ média dos dias de férias anuais da categoria profissional ${ }_{k}$;

$D=$ dias do ano (365 dias).

\section{Ausências não previstas $\left(\mathrm{A}_{\mathrm{k}} \%\right)$}

Esta variável é resultante da soma dos vários tipos de ausências tais como: faltas abonadas, justificadas e injustificadas; licença médica, licença maternidade, licença por acidente de trabalho, licença INSS, outras licenças (nojo, gala, paternidade, etc) e suspensões.

A identificação do percentual deste tipo de ausência foi realizada por meio da identificação das variáveis intervenientes e aplicação da equação( ${ }^{(10)}$ :

$$
A_{k} \%=\left(\frac{\sum_{i} a_{k, i}}{D-\sum_{i} a_{k, i}}\right) \cdot 100
$$

onde:

$k=$ categoria profissional

$A_{k} \%=$ percentual de ausências não previstas, da categoria profissional $_{k}$ (enfermeiro, técnico /auxiliar);

$\sum_{i} a_{k, i}=$ somatória dos dias médios de ausências não previstas, segundo os tipos de ausências (faltas, licenças e suspensões), por categoria profissional ${ }_{k}$;

$\mathrm{D}=$ dias do ano (365 dias).

Para identificar o número médio de dias de ausências por folgas, férias e por todos os outros tipos de ausências não previstas, foi realizado um levantamento mensal do número de dias de cada tipo de ausência, bem como do quantitativo de pessoal existente da Unidade de Pediatria, por categoria profissional (enfermeiras e técnicos/auxiliares de enfermagem), por meio das escalas mensais de trabalho (referentes ao ano 2001) e dos relatórios mensais da Unidade (anos de 2002 e 2005).

No que se refere aos dados dos anos de 2003 e 2004, foram utilizados os percentuais mensais já calculados pelo DE da Instituição. 
Os cálculos referentes à identificação do percentual de ausências previstas e não previstas da equipe de enfermagem da Unidade de Pediatria do HU-USP, foram realizados com auxílio de planilhas eletrônicas.

\section{RESULTADOS}

O levantamento do número de ausências previstas (folga semanal remunerada, folgas por feriados não coincidentes com o domingo e férias) e não previstas (faltas abonadas, justificadas e injustificadas, licença médica, licença maternidade, licença por acidente de trabalho, licença INSS e outras licenças como nojo, gala, paternidade e suspen- sões), permitiu a identificação das médias anuais desses tipos de ausências, apresentadas pelas categorias profissionais envolvidas no estudo, na Unidade de Pediatria, no período de 2001 a 2005.

\section{Ausências previstas por folgas semanais remuneradas e feriados não coincidentes com o domingo $\left(\mathrm{R}_{\mathrm{k}} \%\right)$ e férias $\left(\mathrm{V} \%_{k}\right)$}

Os dados referentes ao percentual médio anual de ausências previstas por folga (semanal remunerada e feriados não coincidentes com o domingo) e férias, por categoria profissional, estão apresentados na Tabela a seguir:

Tabela 1 - Percentual médio anual das ausências previstas por folgas e férias, segundo a categoria profissional, Unidade de Pediatria do HU-USP, período de 2001 a 2005 - São Paulo - 2006

\begin{tabular}{c|cc|cc}
\hline \multirow{2}{*}{ Ausências Previstas } & \multicolumn{2}{|c|}{ Folgas } & \multicolumn{2}{c}{ Férias } \\
\hline Categorias & Enf & T/A & Enf & T/A \\
$\mathbf{2 0 0 1}$ & 18,1 & 19,2 & 6,2 & 6,6 \\
$\mathbf{2 0 0 2}$ & 26,4 & 22,6 & 6,4 & 6,6 \\
$\mathbf{2 0 0 3}$ & 28,1 & 28,3 & 5,4 & 8,5 \\
$\mathbf{2 0 0 4}$ & 27,2 & 23,6 & 7,1 & 7,2 \\
$\mathbf{2 0 0 5}$ & 27,1 & 22,7 & 6,2 & 7,0 \\
\hline Média & 25,4 & 23,3 & 6,3 & \\
\hline
\end{tabular}

Nota: Enf = Enfermeira T/A = Técnico/auxiliar de enfermagem

Observa-se, na Tabela 1, que os percentuais de ausências por folgas (semanais remuneradas e por feriados não coincidentes com o domingo), das categorias enfermeira e técnico/auxiliar de enfermagem, sofreram oscilações durante o período analisado, apresentando maiores percentuais no ano de 2003 (28,1 e 28,3\%, respectivamente). Em relação aos técnicos/auxiliares de enfermagem, as enfermeiras apresentaram maiores percentuais de ausência por folgas em todos os anos, excetuando-se os anos de 2001 e 2003. Este fato pode estar relacionado à realização de horas extras (por necessidade da Unidade ou devido à participação em reuniões administrativas, em grupos de estudo, em programas de treinamento/desenvolvimento realizados, além da jornada diária de trabalho) que, conforme a política da Instituição, não são remuneradas e sim revertidas em folgas.

Estudos realizados ${ }^{(2-8,11-14)}$ em diferentes realidades institucionais, demonstraram que as ausências previstas representam o maior percentual de cobertura, destacando-se o índice referente às folgas. Para uma carga de trabalho de 36 horas semanais, a média de ausências por folga por descanso semanal remunerado corresponde à $16,6 \%$ e por feriados, não coincidentes com o domingo, à $3,4 \%^{(10)}$.

Dessa forma, verifica-se que os percentuais médios de ausências previstas por folga semanal e feriado não coincidente com o domingo encontrados neste estudo, com exceção do ano de 2001, são superiores a soma dos valores indicados $(20 \%)^{(10)}$.
Este fato pode ser justificado pelo número de dias de feriados não coincidentes com os domingos concedidos pela Instituição que, por observar os mesmos dias de feriados facultados pela Universidade a todas as outras Unidades da USP, muitas vezes concede um número maior de feriados do que aqueles normalmente estabelecidos pela lei e decretos federais, estaduais e municipais, como por exemplo, as emendas de feriados.

Os percentuais médios de ausências por férias anuais, da categoria enfermeira e técnicos/auxiliares de enfermagem, da Unidade de Pediatria do HU-USP, apresentou menores índices no ano 2003 (5,4\% e 6,5\%, respectivamente) e maiores no ano de 2004 ( $7,1 \%$ e 8,1\%, respectivamente). Esses percentuais estão em conformidade com os indicados na literatura ${ }^{(10)}$, que apontam, para 20 e 30 dias de férias, os percentuais de $5,8 \%$ e $8,9 \%$, respectivamente.

Comparando-se esses dados com àqueles preconizados pela Resolução $\mathrm{COFEN}^{(9)}$,verifica-se que os percentuais de ausências por férias, apresentado pelos profissionais de enfermagem da Unidade de Pediatria do HU-USP, foram inferiores aos recomendados por esse órgão (8,33\%). Esse fato pode estar relacionado à rotatividade dos profissionais da equipe de enfermagem, bem como com a possibilidade do trabalhador converter $1 / 3$ dos dias de férias em dinheiro (abono pecuniário) e desfrutar somente 20 dias. 


\section{Ausência não previstas (A\%)}

Os dados referentes ao percentual médio de ausências não previstas, bem como sua distribuição entre os diversos tipos (faltas, licença médica, licença maternidade, licença

acidente, licença INSS e outras licenças, como gala, nojo, paternidade e suspensões), por categoria profissional, estão apresentados na tabela a seguir:

Tabela 2 - Percentual médio anual das ausências não previstas, segundo o tipo de ausência e a categoria profissional, Unidade de Pediatria do HU-USP, período de 2001 a 2005 - São Paulo - 2006

\begin{tabular}{|c|c|c|c|c|c|c|c|c|c|c|c|c|c|c|}
\hline $\begin{array}{c}\text { Ausência } \\
\text { não prevista }\end{array}$ & \multicolumn{2}{|c|}{ Faltas } & \multicolumn{2}{|c|}{ Médica } & \multicolumn{2}{|c|}{ Maternidade } & \multicolumn{2}{|c|}{ Acidente } & \multicolumn{2}{|c|}{ INSS } & \multicolumn{2}{|c|}{$\begin{array}{l}\text { Outras } \\
\text { licenças }\end{array}$} & \multicolumn{2}{|c|}{ Total } \\
\hline Categorias & ENF & T/A & ENF & T/A & ENF & T/A & ENF & T/A & ENF & T/A & ENF & T/A & ENF & T/A \\
\hline 2001 & 0,5 & 0,3 & 1,0 & 0,7 & 0,0 & 0,5 & 0,0 & 0,1 & 0,0 & 2,4 & 0,0 & 0,1 & 1,5 & 4,1 \\
\hline 2002 & 0,3 & 0,2 & 0,5 & 0,3 & 0,3 & 0,0 & 0,0 & 0,0 & 1,4 & 0,0 & 0,0 & 0,0 & 2,5 & 0,5 \\
\hline 2003 & 0,1 & 0,1 & 1,2 & 0,8 & 0,0 & 1,3 & 0,1 & 0,0 & 0,0 & 0,0 & 0,1 & 0,1 & 1,5 & 2,3 \\
\hline 2004 & 0,0 & 0,2 & 1,0 & 2,2 & 4,9 & 1,1 & 0,0 & 0,0 & 0,0 & 0,0 & 0,2 & 0,0 & 6,1 & 3,7 \\
\hline 2005 & 0,7 & 0,1 & 1,2 & 0,8 & 2,8 & 1,2 & 0,0 & 0,0 & 0,0 & 4,5 & 0,0 & 0,1 & 4,7 & 6,7 \\
\hline Média & 0,3 & 0,2 & 1,0 & 1,0 & 1,6 & 0,8 & 0,0 & 0,0 & 0,3 & 1,4 & 0,1 & 0,1 & 3,3 & 3,5 \\
\hline
\end{tabular}

Nota: Enf = Enfermeira T/A = Técnico/auxiliar de enfermagem

De acordo com a Tabela 2, verifica-se, com relação à categoria enfermeira, predomínio de ausências não previstas por licença maternidade e licença médica. Nos anos de 2001 e 2003, os maiores percentuais estiveram relacionados à licença médica (1,0\% e 1,2\%, respectivamente). Em 2002, o maior índice de ausência não prevista foi resultante da licença INSS $(1,4 \%)$, não verificada nos demais períodos. Nos anos de 2004 e 2005, a licença maternidade foi responsável pelos índices mais elevados de ausências não previstas da categoria ( $4,9 \%$ e $2,8 \%$, respectivamente).

No que se refere à categoria técnico/auxiliar de enfermagem, as ausências não previstas por licença INSS e licença médica representaram os maiores índices de ausências não previstas no período do estudo. Assim, observa-se que nos anos de 2001 e 2005 a licença INSS representou os maiores percentuais desse tipo de ausência (2,4\% e 4,5\%, respectivamente). No ano 2002 a licença médica (0,3\%) representou o maior índice, embora esse valor apresente pouco significado; nos anos de 2003 e 2004 as licenças maternidade e médica foram responsáveis pelos maiores índices de ausências não previstas da categoria (1,3\% e 2,2\%, respectivamente).

Estudos desenvolvidos por vários autores ${ }^{(6-8,13)}$ indicam a licença médica como o principal motivo de ausências não previstas da equipe de enfermagem. Na presente pesquisa constata-se que esse tipo de ausência constituiu-se um dos princi-pais motivos de ausências não previstas dos trabalhadores de enfermagem, no entanto não representou o principal motivo.

Os dados referentes às ausências não previstas por licença maternidade, encontrados neste estudo, traduzem o perfil dos integrantes da equipe de enfermagem da Unidade de Pediatria e corroboram os resultados de outro estudo ${ }^{(12)}$, desenvolvido na mesma Instituição, que descreve a equipe de enfermagem do HU-USP como um grupo jovem, predominantemente do sexo feminino.

Analisando-se os percentuais totais de ausências não previstas, encontrados em cada ano, observa-se que os percentuais apresentados tanto pelas enfermeiras quanto pelos técnicos/auxiliares de enfermagem, excetuando-se os apresentados pelos técnicos/auxiliares de enfermagem, no ano de 2005, foram inferiores ao percentual indicado pelo COFEN $^{(9)}$ para a cobertura desse tipo de ausência (6,67\%).

Diante dos resultados encontrados, referentes às ausências previstas e não previstas, calculou-se o índice de segurança técnica de cada categoria profissional, obtido por meio do produto de todos os tipos de ausências, conforme demonstrado na equação apresentada anteriormente. Os resultados encontrados estão sintetizados na Tabela 3.

Tabela 3 - Índice de Segurança Técnica, segundo a categoria profissional, Unidade de Pediatria do HU-USP, período de 2001 a 2005 - São Paulo - 2006

\begin{tabular}{cccccccccccc}
\hline Ano & \multicolumn{2}{c}{$\mathbf{2 0 0 1}$} & \multicolumn{2}{c}{$\mathbf{2 0 0 2}$} & \multicolumn{2}{c}{$\mathbf{2 0 0 3}$} & \multicolumn{2}{c}{$\mathbf{2 0 0 4}$} & \multicolumn{2}{c}{$\mathbf{2 0 0 5}$} \\
\hline Categoria & Enf & T/A & Enf & T/A & Enf & T/A & Enf & T/A & Enf & T/A \\
\hline \multirow{2}{*}{ IST } & 27,4 & 32,2 & 37,5 & 31,8 & 37,0 & 39,8 & 44,7 & 38,5 & 41,3 & 40,4 \\
\hline
\end{tabular}

Nota: Enf = Enfermeira T/A = Técnico/auxiliar de enfermagem 
Observa-se na tabela 3, que o IST da Unidade de Pediatria do HU-USP sofreu variações ao longo do período analisado, apresentando os maiores índices nos anos de 2004 (categoria enfermeira) e 2005 (categoria técnico/auxiliar de enfermagem). $\mathrm{O}$ tipo de ausência que mais contribuiu para a elevação desse índice está relacionado com o número de folgas da equipe de enfermagem, corroborando a constatação de outros pesquisadores, conforme citado anteriormente.

Comparando-se esses dados com os encontrados em outro estudo ${ }^{(12)}$, realizado no HU-USP no ano 2000, verifica-se que os IST da categoria técnico/auxiliar de enfermagem, da Unidade de Pediatria, no período de 2001 a 2005, foram inferiores aos encontrados no ano 2000 (49\%). Entretanto, os IST da categoria enfermeira, nos anos de 2002, 2004 e 2005, foram superiores ao encontrado naquele período (37\%).

A análise desses dados evidencia a importância de serem realizadas avaliações sistemáticas das ausências da equi-pe de enfermagem, principalmente, no que se refere à utilização dessas informações como subsídio para a avaliação da carga de trabalho e, consequentemente, do quadro de pessoal das unidades de internação de instituições hospitalares.

\section{CONCLUSÕES}

A identificação do percentual de ausências previstas e não previstas da equipe de enfermagem da Unidade de Pediatria do HU-USP possibilitou identificar o IST da Unidade, no período de 2001 a 2005, bem como permitiu melhor compreensão dos principais fatores que interferem na sua determinação.

\section{REFERÊNCIAS}

1. Fugulin MFT, Gaidzinski RR, Kurcgant P. Ausências previstas e não previstas da equipe de enfermagem das unidades de internação do HU-USP. Rev Esc Enferm USP. 2003;37(4): 109-17.

2. Gaidzinski RR, Sanna MC, Leite MMJ, Mayor ERC. Estudo das ausências da equipe de enfermagem num hospital geral de grande porte. Rev Enferm Complexo HC/FMUSP. 1998;1(4):8-14.

3. Laus AM. Dimensionamento de pessoal de enfermagem para unidades de internação médicas e cirúrgicas no Hospital das Clínicas da Faculdade de Medicina de Ribeirão Preto - USP [tese]. Ribeirão Preto: Escola de Enfermagem de Ribeirão Preto, Universidade de São Paulo; 2003.

4. Fugulin FMT. Sistema de classificação de pacientes: análise das horas de assistência de enfermagem [dissertação]. São Paulo: Escola de Enfermagem, Universidade de São Paulo; 1997.
Assim, verificou-se que o percentual de ausências por folga correspondeu ao maior percentual de cobertura dos profissionais de enfermagem, corroborando os dados apontados em outros estudos sobre a temática ${ }^{(1-8,11-14)}$, evidenciando, ainda, que a necessidade de realização de horas extras, quando não remuneradas e sim revertidas em folgas pela instituição, pode contribuir para elevar este percentual.

No que se refere às ausências previstas por feriados não coincidentes com o domingo e férias, observou-se que esses percentuais variam de acordo com o número de feriados concedidos pela instituição e com a possibilidade do trabalhador converter $1 / 3$ dos dias de férias em dinheiro (abono pecuniário) e desfrutar somente 20 dias.

O levantamento do percentual de ausências não previstas da equipe de enfermagem da Unidade de Pediatria mostrou uma variação entre os índices encontrados em cada ano e identificou as licenças maternidade, INSS e médica como os principais motivos de ausências não previstas dos profissionais de enfermagem da Unidade, no período. Analisando-se os percentuais totais de ausências não previstas, observa-se que esses percentuais são inferiores àqueles referentes às ausências previstas por folgas e férias. Essa constatação contraria o discurso de muitas enfermeiras que relacionam esse tipo de ausência como o principal responsável pela insuficiência de quantitativo de pessoal de enfermagem nas instituições de saúde.

A variação dos IST, evidenciados no período de 2001 a 2005, apontou a importância de serem realizadas avaliações sistemáticas das ausências da equipe de enfermagem, assim como de se identificarem índices específicos das unidades, como subsídio para a avaliação do quadro de pessoal de enfermagem nas organizações de saúde.

5. Belém IJR, Gaidzinski RR. Estudo das ausências da equipe de enfermagem. Rev Bras Enferm. 1998;51(4):697-708.

6. Farias FAC, Silva AL, Gaidzinski RR. Dimensionamento de pessoal de enfermagem: análise em um hospital de cardiologia. In: Anais do $7^{\circ}$ Encontro de Enfermagem e Tecnologia; 2000; São Paulo [CD-ROM]. São Paulo: ENFTEC; 2000.

7. Pavani LMD. Análise do dimensionamento de pessoal de enfermagem na Unidade de Pós-operatório de Cirurgia Cardíaca em um Hospital Universitário especializado em cardiologia [dissertação]. São Paulo: Escola de Enfermagem, Universidade de São Paulo; 2000.

8. Possari JF. Dimensionamento de pessoal de enfermagem em centro cirúrgico no período transoperatório: estudo das horas de assistência, segundo o porte cirúrgico [dissertação]. São Paulo: Escola de Enfermagem, Universidade de São Paulo; 2001. 
9. Conselho Federal de Enfermagem (COFEn). Resolução n. 293, de 21 de setembro de 2004. Fixa e estabelece parâmetros para dimensionamento do quadro de profissionais de enfermagem nas unidades assistenciais das instituições de saúde e assemelhados [legislação na Internet]. Rio de Janeiro; 2004 [citado 2006 jul. 14]. Disponível em: http://corensp.org.br/072005/legislacoes/ legislacoes_busca.php?leg_id $=10105 \&$ texto $=$

10. Gaidzinski RR. O dimensionamento do pessoal em instituições hospitalares [tese]. São Paulo: Escola de Enfermagem, Universidade de São Paulo; 1998.

11. Matsushita MS. Dimensionamento do pessoal de enfermagem das Unidades de Internação do Hospital São Paulo [dissertação]. São Paulo: Escola de Enfermagem, Universidade de São Paulo; 2003.
12. Fugulin MFT. Dimensionamento de pessoal de enfermagem: avaliação do quadro de pessoal das unidades de internação de um hospital de ensino [tese]. São Paulo: Escola de Enfermagem, Universidade de São Paulo; 2002.

13. Farias FAC. Dimensionamento de pessoal de enfermagem para o Pronto Socorro especializado em afecções cardiovasculares [tese]. São Paulo: Escola de Enfermagem, Universidade de São Paulo; 2003.

14. Nascimento GM. Estudo do absenteísmo dos trabalhadores de enfermagem em uma Unidade Básica e Distrital de Saúde do Município de Ribeirão Preto-SP [dissertação]. Ribeirão Preto: Escola de Enfermagem de Ribeirão Preto, Universidade de São Paulo; 2003. 